\title{
Risk-averse asymptotics for reservation prices
}

\author{
Laurence Carassus * $^{*} \quad$ Miklós Rásonyi ${ }^{\dagger}$
}

November 1, 2018

\begin{abstract}
An investor's risk aversion is assumed to tend to infinity. In a fairly general setting, we present conditions ensuring that the respective utility indifference prices of a given contingent claim converge to its superreplication price.
\end{abstract}

Keywords: Utility indifference price, Superreplication price, Convergence, Utility maximization, Risk aversion.

MSC 2000 Subject Classification: Primary: 91B16, 91B28 ; Secondary: 93E20, 49L20

OR/MS Subject Classification: Primary: Utility / Value theory ; Secondary: Finance / Asset Pricing

JEL classification: C61, C62, G11, G12

\section{Introduction}

In this article we investigate the effect of increasing risk aversion on utilitybased prices. We are dealing with the utility indifference price (or reservation price), defined in 12 for the first time. This is the minimal amount added to an option seller's initial capital which allows her to attain the same utility that she would have attained from her initial capital without selling the option, see Definition 4.2 below. Intuitively, when risk aversion tends to infinity, reservation price should tend to the superreplication price (i.e. the price of hedging the option without any risk).

This result was shown in 19 for Brownian models and in 9 in a semimartingale setting when the agent has constant absolute risk aversion (i.e. for exponential utility functions). Certain other classes of utility functions were treated in [4, models with transaction costs were considered in [5].

However, an extension of this result to general utility functions was lacking. In $[6$ and $[7$ the case of discrete-time markets was treated for utilities on the positive axis as well as on the real line. Now we prove this result in a continuoustime semimartingale framework, under suitable hypotheses.

\footnotetext{
* Laboratoire de Probabilités et Modèles Aléatoires, Université Paris 7 Denis Diderot, 16 rue Clisson, 75013 Paris, France (e-mail: carassus@math.jussieu.fr)

${ }^{\dagger}$ The Computer and Automation Institute of the Hungarian Academy of Sciences, 1518 Budapest, P. O. Box 63., Hungary (e-mail: rasonyi@sztaki.hu). The second author acknowledges financial support from the Hungarian Science Foundation (OTKA) grant F 049094 and dedicates this paper to Annamária Brecz.
} 
In section 2 we model the agent's preferences and introduce a growth condition (related to the elasticity of utility functions), in section 3 the market model and a compactness assumption are discussed. In section 4 the concept of utility indifference price is formally defined and the two main theorems are proved.

\section{Risk averse agents}

We consider agents trading in the market with initial endowment $z \in \mathbb{R}$. We assume that

Assumption 2.1 $U_{n}, n \in \mathbb{N}$ are twice continuously differentiable, strictly concave and increasing functions on $\mathbb{R}$ such that for each $x \in \mathbb{R}$,

$$
r_{n}(x):=\frac{-U_{n}^{\prime \prime}(x)}{U_{n}^{\prime}(x)} \underset{n \rightarrow+\infty}{\longrightarrow} \infty .
$$

The function $r_{n}$ is called the (absolute) risk aversion of an agent with utility function $U_{n}$. This concept was introduced in [1] and [17. Here we are interested in the case where this measure of risk aversion tends to infinity.

We take the Fenchel conjugates of $U_{n}$ :

$$
V_{n}(y):=\sup _{x \in \mathbb{R}}\left\{U_{n}(x)-x y\right\}, \quad y \in(0, \infty) .
$$

As easily checked, $V_{n}$ is a finite convex function.

We stipulate a growth condition on the conjugates of the utility functions we consider. Such assumptions are often referred to as "elasticity conditions".

Assumption 2.2 For each $\left[\lambda_{0}, \lambda_{1}\right] \subset(0, \infty)$ there exist positive constants $C_{1}, C_{2}, C_{3}$ such that for all $n$ and for all $y>0$,

$$
V_{n}(\lambda y) \leq C_{1} V_{n}(y)+C_{2} y+C_{3} .
$$

holds for each $\lambda \in\left[\lambda_{0}, \lambda_{1}\right]$.

Remark 2.3 For $n$ fixed, condition (3) is equivalent to

$$
\limsup _{x \rightarrow \infty} \frac{x U_{n}^{\prime}(x)}{U_{n}(x)}<1, \quad \liminf _{x \rightarrow-\infty} \frac{x U_{n}^{\prime}(x)}{U_{n}(x)}>1,
$$

as shown in [11.

The first of the two conditions in (4) was introduced in [15, the second one in [20. A utility function $U_{n}$ satisfying (4) is said to have reasonable asymptotic elasticity (terminology of 20). Thus condition (3) is a (dually formulated) uniform reasonable asymptotic elasticity condition. Another uniform asymptotic elasticity condition appears as Assumption 2.3 of $[8$. About the derivation of equivalances like that of (4) and (3) consult section 6 of [15], section 4 of [20] and [11. 


\section{Market Model}

Our market is modelled by an adapted $d$-dimensional stochastic process $S$ on a given continuous-time stochastic basis $\left(\Omega, \mathcal{F},\left(\mathcal{F}_{t}\right)_{t \geq 0}, P\right)$. We think that $S$ represents the evolution of the (discounted) prices of $\bar{d}$ assets. For simplicity we assume $S$ locally bounded (to avoid technical complications related to $\sigma$ martingales). Absence of arbitrage is stipulated by

$$
\mathcal{M} \neq \emptyset
$$

where $\mathcal{M}$ denotes the set of measures $Q \sim P$ such that $S$ is a local martingale under $Q$.

We make the following compactness assumption.

Assumption 3.1 There exists $Q_{0} \in \mathcal{M}$ such that the sequence $V_{n}\left(d Q_{0} / d P\right)$ is uniformly integrable (with respect to $P$ ). We denote by $\mathcal{M}_{v}$ the set of such $Q_{0} \mathrm{~s}$.

Remark 3.2 Similar conditions have already appeared in investigations on the stability of optimal strategies with respect to perturbations of utility functions, see 16 and [14. Both papers consider a sequence $U_{n}$ of utility functions converging to a limiting utility $U$ and show convergence of the corresponding optimal strategies/utility prices.

In 16 a sequence $U_{n}$ is assumed to be dominated by some $\bar{U}$ with conjugate function $\bar{V}$ such that $\bar{V}(Z)$ is integrable, where $Z$ is (the candidate for) the density of the minimal martingale measure. In Assumption (UI) of 14 we find essentially the uniform integrability of $V_{n}\left(y d Q_{n} / d P\right)$ for each $y>0$, where $Q_{n}$ is a suitable sequence in $\mathcal{M}$. Note that 14 also considers perturbations of the underlying probability measure and that in both mentioned papers the $U_{n}$ are defined on the positive axis.

This elasticity hypothesis 2.2 allows us to prove that the set $\mathcal{M}_{v}$ is in fact large.

Lemma 3.3 Assume that there is $x_{0} \in \mathbb{R}$ such that $U_{n}\left(x_{0}\right)$ is bounded from below. Under Assumptions 2.2 and 3.1 the set $\mathcal{M}_{v}$ is dense in $\mathcal{M}$ with respect to the total variation norm topology.

Proof. This result was essentially reported in Proposition 6 of [3], without proof. Fix $Q_{0} \in \mathcal{M}_{v}$. We know from 13 that the set

$$
\mathcal{M}_{b}:=\left\{Q \in \mathcal{M}: d Q / d Q_{0} \text { is bounded }\right\}
$$

is dense in $\mathcal{M}$. It follows by concavity of $\mathcal{M}$ that also

$$
\mathcal{M}_{b b}:=\left\{\alpha Q_{0}+(1-\alpha) Q: 0<\alpha<1, Q \in \mathcal{M}_{b}\right\}
$$

is a dense subset of $\mathcal{M}$. We shall show that $\mathcal{M}_{b b} \subset \mathcal{M}_{v}$. Take an arbitrary $Q \in \mathcal{M}_{b}$ such that $d Q / d Q_{0} \leq K$ and any fixed $0<\alpha<1$. We have

$V_{n}\left(\alpha d Q_{0} / d P+(1-\alpha) d Q / d P\right) \geq U_{n}\left(x_{0}\right)-x_{0}\left(\alpha d Q_{0} / d P+(1-\alpha) d Q / d P\right)$.

Moreover,

$$
V_{n}\left(\alpha d Q_{0} / d P+(1-\alpha) d Q / d P\right) \leq C_{1} V_{n}\left(d Q_{0} / d P\right)+C_{2}\left(d Q_{0} / d P\right)+C_{3}
$$


by Assumption 2.2 (choose $C_{1}, C_{2}, C_{3}$ corresponding to the interval $[\alpha, \alpha+(1-$ $\alpha) K]$ and note that $\alpha+(1-\alpha) d Q_{0} / d Q$ falls into this interval).

Uniform integrability of $V_{n}\left(\alpha d Q_{0} / d P+(1-\alpha) d Q / d P\right), n \in \mathbb{N}$ now follows from Assumption 3.1 .

\section{Utility indifference prices}

It follows from $\mathcal{M} \neq \emptyset$ that $S$ is a semimartingale and we may define the set of admissible trading strategies as predictable $S$-integrable processes with a finite credit line (to avoid doubling strategies).

\section{Definition 4.1}

$\mathcal{A}:=\left\{\phi: \phi\right.$ predictable, $S$-integrable and for some $\left.w>0, V^{0, \phi} \geq-w\right\}$,

where we write $V_{t}^{x, \phi}:=x+(\phi \cdot S)_{t}$ for the value process of strategy $\phi$ starting from initial endowment $x$.

Fix $T>0$ and a bounded random variable $G$, interpreted as a contingent claim to be delivered at the end of the period $[0, T]$. By optimal trading an agent with initial capital $x \in \mathbb{R}$ and utility $U_{n}$ delivering the claim $G$ may attain

$$
u_{n}(x, G):=\sup _{\phi \in \mathcal{A}} E U_{n}\left(V_{T}^{x, \phi}-G\right),
$$

this is well-defined as admissible strategies have bounded from below value processes.

Definition 4.2 The utility indifference price for $U_{n}$ and initial endowment $z$ is

$$
p_{n}(z, G):=\inf \left\{p \in \mathbb{R}: u_{n}(z+p, G) \geq u_{n}(z, 0)\right\},
$$

the minimal extra capital that allows for delivering $G$ while attaining the same utility as without claim delivery.

The utility-free concept of the superhedging price of a bounded contingent claim (random variable) $G$ is defined as

$$
\pi(G):=\inf \left\{x \in \mathbb{R}: \text { there is } \phi \in \mathcal{A} \text { such that } V_{T}^{x, \phi} \geq G \text { a.s. }\right\} .
$$

The following dual characterization is a fundamental result of mathematical finance, see e.g. [10] and the references therein.

Theorem 4.3 Under $\mathcal{M} \neq \emptyset$, we have

$$
\pi(G)=\sup _{Q \in \mathcal{M}} E_{Q} G .
$$

We are now ready to state our main result i.e. the convergence of utility indifference prices to the superreplication price. We will present this result under two types of assumptions. The first one (see Assumption 4.4) refers to the existence of a initial wealth for which all the investors have (asymptotically) a common preference for $x_{0}$ and also a common non zero growth rate for their preferences near $x_{0}$.

The second kind of assumptions impose the elasticity Assumption 2.2 and the compactness Assumption 3.1 on a normalized family of utility functions (see Theorem 4.13). 
Assumption 4.4 There exists some $x_{0}, \alpha, \beta \in \mathbb{R}$ with $\alpha \in(0, \infty)$ and $|\beta|<\infty$ such that :

$$
\begin{array}{lll}
U_{n}^{\prime}\left(x_{0}\right) & \underset{n \rightarrow+\infty}{\longrightarrow} & \alpha \\
U_{n}\left(x_{0}\right) & \underset{n \rightarrow+\infty}{\longrightarrow} & \beta
\end{array}
$$

Remark 4.5 We will see in the examples below that this assumption is satisfied for power and exponential utility functions. In those examples, we have that $U_{n}(0)=0$ and $U_{n}^{\prime}(0)=1$. This means that all investors consider that utility of nothing is zero and that for very small wealth their utility functions are approximatively linear with slope one.

The following proposition is the first step to Theorem 4.7:

Proposition 4.6 Under Assumptions 2.1 and 4.4, for each $y>0$,

$$
V_{n}(y) \underset{n \rightarrow+\infty}{\longrightarrow} \beta-x_{0} y \text {. }
$$

Proof. First, the argument of Lemma 4 in [6] shows that as $n \rightarrow \infty$,

$$
U_{n}^{\prime}(x) \rightarrow \infty, x<x_{0}, \quad U_{n}^{\prime}(x) \rightarrow 0, x>x_{0} .
$$

Let $I_{n}$ be the inverse of $U_{n}^{\prime}$ (which exists by strict concavity of $U_{n}$ ). We claim

$$
I_{n}(y) \rightarrow x_{0}, \text { for } y>0 .
$$

Indeed, let $y>0$. If we had $I_{n_{k}}(y) \geq x_{0}+\varepsilon$ for some $\varepsilon>0$ and a subsequence $n_{k}$ then $U_{n_{k}}^{\prime}\left(I_{n_{k}}(y)\right)=y \leq U_{n_{k}}^{\prime}\left(x_{0}+\varepsilon\right)$, but this latter tends to 0 by (7), a contradiction. The proof of the reverse inequality is similar and $I_{n}(y) \rightarrow x_{0}$.

First by definition $V_{n}(y) \geq U_{n}\left(x_{0}\right)-x_{0} y$. Since $V_{n}$ can be calculated as $V_{n}(y)=U_{n}\left(I_{n}(y)\right)-I_{n}(y) y$; concavity of $U_{n}$ implies that $V_{n}(y) \leq U_{n}\left(x_{0}\right)+$ $\left(I_{n}(y)-x_{0}\right) U_{n}^{\prime}\left(x_{0}\right)-I_{n}(y) y$. Thus

$$
0 \leq V_{n}(y)-\left(U_{n}\left(x_{0}\right)-x_{0} y\right) \leq\left(I_{n}(y)-x_{0}\right)\left(U_{n}^{\prime}\left(x_{0}\right)-y\right) \rightarrow 0,
$$

$n \rightarrow \infty$, showing the claim.

Theorem 4.7 If Assumptions 2.1, 2.2, 3.1 and 4.4 hold then for each bounded random variable $G$ the quantities $u_{n}\left(x_{0}, G\right), n \in \mathbb{N}$ are finite and the corresponding utility indifference prices $p_{n}\left(x_{0}, G\right)$ tend to $\pi(G)$ as $n \rightarrow \infty$.

Remark 4.8 The convergence in Theorem 4.7 holds only for $x_{0}$. We will see in Theorem 4.13 how the convergence for all initial capital $z$ can be checked under a second type of assumptions.

Proof. It is a standard fact that that $p_{n}\left(x_{0}, G\right) \leq \pi(G)$, see page 152 of [6]. For the reverse inequality, we argue by contradiction. Suppose that for some $\varepsilon>0$ and a subsequence $n_{k}$ one has $p_{k}:=p_{n_{k}}\left(x_{0}, G\right) \leq \pi(G)-\varepsilon$ for each $k$. We may suppose that $n_{k}=k$ and $p_{k} \rightarrow \pi(G)-\varepsilon, k \rightarrow \infty$. 
Take arbitrary $\phi \in \mathcal{A}$ and $Q \in \mathcal{M}_{v}$. Then $E_{Q}\left(V_{T}^{x_{0}+p_{k}, \phi}-G\right) \leq x_{0}+p_{k}-E_{Q} G$ $\left(V^{0, \phi}\right.$ is a supermartingale by results of [2]). Proposition 4.6 and $Q \in \mathcal{M}_{v}$ imply

$$
\begin{array}{r}
\limsup _{k \rightarrow \infty} E\left(V_{k}(d Q / d P)+(d Q / d P)\left(V_{T}^{x_{0}+p_{k}, \phi}-G\right)\right) \\
\leq \beta-x_{0}+\left(x_{0}+\limsup _{k \rightarrow \infty} p_{k}-E_{Q} G\right) .
\end{array}
$$

Introduce

$$
v_{k}^{\phi}:=\inf _{Q \in \mathcal{M}_{v}} E\left(V_{k}(d Q / d P)+(d Q / d P)\left(V_{T}^{x_{0}+p_{k}, \phi}-G\right)\right) .
$$

It follows from Lemma 3.3 that $\sup _{Q \in \mathcal{M}} E_{Q} G=\sup _{Q \in \mathcal{M}} E_{Q} G$, thus (8) and Theorem 4.3 imply

$$
\limsup _{k \rightarrow \infty} v_{k}^{\phi} \leq \beta+\lim _{k \rightarrow \infty} p_{k}-\pi(G)=\beta-\varepsilon .
$$

By the definition of conjugate functions we have,

$$
\limsup _{k \rightarrow \infty} E U_{k}\left(V_{T}^{x_{0}+p_{k}, \phi}-G\right) \leq \limsup _{k \rightarrow \infty} v_{k}^{\phi} \leq \beta-\varepsilon .
$$

It follows that $u_{k}$ are finite and $\lim \sup _{k \rightarrow \infty} u_{k}\left(x_{0}+p_{k}, G\right) \leq \beta-\varepsilon$. But $u_{k}\left(x_{0}+p_{k}, G\right) \geq u_{k}\left(x_{0}, 0\right) \geq U_{k}\left(x_{0}\right)$, thus $\limsup _{k \rightarrow \infty} u_{k}\left(x_{0}+p_{k}, G\right) \geq \beta$ a contradiction.

Remark 4.9 Let us consider the condition that there exists $Q_{0} \in \mathcal{M}$ such that $d Q_{0} / d P$ and $d P / d Q_{0}$ are both bounded (by some $K>0$ ).

Note that a convex function attains its maximum on an interval at one of the endpoints. Hence

$$
U_{n}\left(x_{0}\right)-\left|x_{0}\right| K \leq V_{n}\left(d Q_{0} / d P\right) \leq\left|V_{n}(K)\right|+\left|V_{n}(1 / K)\right|
$$

which is bounded by Proposition 4.6, showing that this condition is stronger that Assumption 3.1.

We claim that, replacing Assumption 3.1 by this condition, one may drop Assumption 2.2 from the hypotheses of Theorem 4.7. Indeed, the result of [13] directly implies that measures $Q_{0}$ with the above property are dense in $\mathcal{M}$, without appeal to Assumption 2.2. The rest of the proof is identical.

Unfortunately, in continuous-time models one rarely finds such a $Q_{0}$. In discrete-time models, however, such measures often exist, see [18] for an extensive discussion.

We now turn to our second Theorem. To state its hypothesis, we first need to introduce some normalization of the functions $U_{n}$. Fix some initial wealth $z \in \mathbb{R}$ and set :

$$
\tilde{U}_{n}(x):=\frac{U_{n}(x)-U_{n}(z)}{U_{n}^{\prime}(z)}, \quad n \in \mathbb{N}, x \in \mathbb{R} .
$$

We now restate Assumptions 2.2 and 3.1 for the family $\left(\tilde{U}_{n}\right)_{n}$.

Assumption 4.10 For each $\left[\lambda_{0}, \lambda_{1}\right] \subset(0, \infty)$ there exist constants $C_{1}, C_{2}, C_{3}$ such that for all $n$ and for all $y>0$,

$$
\tilde{V}_{n}(\lambda y) \leq C_{1} \tilde{V}_{n}(y)+C_{2} y+C_{3} .
$$

holds for each $\lambda \in\left[\lambda_{0}, \lambda_{1}\right]$. 
Assumption 4.11 There exists $Q_{0} \in \mathcal{M}$ such that the sequence $\tilde{V}_{n}\left(d Q_{0} / d P\right)$ is uniformly integrable (with respect to $P$ ).

Remark 4.12 We think that Assumptions 4.10 and 4.11 should be considered as technical assumptions to be checked on $\tilde{U}_{n}$. We will see below that they hold true in our examples.

Theorem 4.13 Assume that Assumptions 2.1, 4.10 and 4.11 hold. Then for each bounded random variable $G$ the indifference prices $p_{n}(z, G)$ tend to $\pi(G)$ as $n \rightarrow \infty$.

Proof. Obviously, $\tilde{U}_{n}(z)=0$ and $\tilde{U}_{n}^{\prime}(z)=1$, thus Assumption 4.4 is satisfied for the sequence $\tilde{U}_{n}$ at $x_{0}=z$. Let $\tilde{u}_{n}$ be defined analogously as in (5), with $\tilde{U}_{n}$ replacing $U_{n}$. Then since the $\tilde{U}_{n}$ are affine transforms of the $U_{n}$, we may alternatively write

$$
p_{n}(z, G)=\inf \left\{p \in \mathbb{R}: \tilde{u}_{n}(z+p, G) \geq \tilde{u}_{n}(z, 0)\right\} .
$$

It is obvious that if Assumption 2.1 hold for $U_{n}$ then it holds also for $\tilde{U}_{n}$, so Theorem 4.7 applied for $\tilde{U}_{n}$ and $x_{0}=z$ allow us to conclude.

Example 4.14 (The exponential case) Let

$$
U_{n}(x)=\frac{1-\exp \left\{-\alpha_{n} x\right\}}{\alpha_{n}}
$$

with some $\alpha_{n}>0$ tending to $\infty$ as $n \rightarrow \infty$. It is straightforward that $U_{n}(0)=$ $0, U_{n}^{\prime}(0)=1, r_{n}(x)=\alpha_{n}$ and thus $U_{n}$ satisfy Assumption 2.1 and 4.4 for $x_{0}=0$. Moreover, calculation gives $V_{n}(y)=\left(1 / \alpha_{n}\right)[y \ln y+1-y]$ showing that Assumption 3.1 holds provided that there is $Q_{0} \sim P$ with $E_{Q_{0}} \ln \left(d Q_{0} / d P\right)<\infty$, i.e. whenever a finite-entropy martingale measure exists. It is enough to show (3) for one function, namely $V(y)=y \ln y$. This is trivial since $\lambda y \ln (\lambda y) \leq$ $\lambda y \ln y+\lambda|\ln \lambda| y$ for $\lambda, y>0$.

So Theorem 4.7 applies and the respective reservation prices $p_{n}(0, G)$ converge to $\pi(G)$.

In fact, one can check that Theorem 4.13 applies for each $z \in \mathbb{R}$. We have thus retrieved the result in $[9$. (They make a weaker moment assumption on $G$; this extension follows by our method, too.)

Example 4.15 (The power case)

$$
U_{n}(x)=-\frac{1}{\alpha_{n}}\left[(x+1)^{-\alpha_{n}}-1\right] 1_{\{x>0\}}-\frac{1}{\beta_{n}}\left[(1-x)^{\beta_{n}}-1\right] 1_{\{x \leq 0\}}
$$

with $\alpha_{n}>0, \beta_{n}>1$ tending to $\infty$. These functions are continuously differentiable with strictly monotone derivatives, hence they are strictly concave. They are also twice continuously differentiable on $\mathbb{R} \backslash\{0\}$ and satisfy (1) on $\mathbb{R} \backslash\{0\}$. Take now $\beta_{n}:=\alpha_{n}+2$ then $U_{n}^{\prime \prime}$ exists and (1) holds in 0 , too.

$$
\begin{aligned}
& U_{n}^{\prime}(x)=(x+1)^{-\left(\alpha_{n}+1\right)} 1_{\{x>0\}}-(1-x)^{\alpha_{n}+1} 1_{\{x<0\}} \\
& \left.U_{n}^{\prime \prime}(x)=-\left(\alpha_{n}+1\right)(x+1)^{-\left(\alpha_{n}+2\right)} 1_{\{x>0\}}-\left(\alpha_{n}+1\right)(1-x)^{\alpha_{n}} 1_{\{x<0\}}\right\}
\end{aligned}
$$


Thus $U_{n}(0)=0, U_{n}^{\prime}(0)=1, r_{n}(x)=\left(\alpha_{n}+1\right)\left(\frac{1_{\{x>0\}}}{x+1}+\frac{1_{\{x<0\}}}{1-x}\right)$ and $U_{n}$ satisfy Assumptions 2.1 and 4.4 for $x_{0}=0$.

It remains to check (3) and Assumption 3.1. We have, for $y \in(0, \infty)$,

$V_{n}(y)=\left[\frac{1}{\alpha_{n}+2}-y+\left(1-\frac{1}{\alpha_{n}+2}\right) y^{\frac{\alpha_{n}+2}{\alpha_{n}+1}}\right] 1_{\{y>1\}}+\left[\frac{1}{\alpha_{n}}+y-\left(1+\frac{1}{\alpha_{n}}\right) y^{\frac{\alpha_{n}}{\alpha_{n}+1}}\right] 1_{\{y \leq 1\}}$.

Thus if there exists $Q_{0} \in \mathcal{M}$ such that $d Q_{0} / d P \in L^{1+\varepsilon}$ for some $\varepsilon>0$ then Assumption 3.1 will be satisfied for $n$ large enough since $\left(\alpha_{n}+2\right) /\left(\alpha_{n}+1\right)$ converges to 1 . Finally, (3) is trivially satisfied by the scaling properties of the power functions. We may conclude by Theorem 4.7 that the reservation prices $p_{n}(0, G)$ tend to $\pi(G)$ as $n \rightarrow \infty$.

Tedious calculations show that we get the same conclusion for any initial capital $z \in \mathbb{R}$ and we may apply Theorem 4.13 .

\section{References}

[1] K. Arrow. Essays in the Theory of Risk-Bearing. North-Holland, Amsterdam, 1965.

[2] J.-P. Ansel, Ch. Stricker. Couverture des actifs contingents et prix maximum. Annales de l'Institut Henri Poincaré. Probabilités et Statistiques, $\mathbf{3 0}$, 303-315, 1994.

[3] S. Biagini, M. Frittelli. On the super-replication price of unbounded claims. Annals of Applied Probability, 14, 1970-1991, 2004.

[4] B. Bouchard. Stochastic control and applications in finance. PhD thesis, Université Paris 9, 2000.

[5] B. Bouchard, Yu. M. Kabanov, N.Touzi. Option pricing by large risk aversion utility under transaction costs. Decis. Econ. Finance, 24, 127-136, 2001.

[6] L. Carassus, M. Rásonyi. Convergence of utility indifference prices to the superreplication price. Mathematical Methods of Operations Research, 64, 145-154, 2006.

[7] L. Carassus, M. Rásonyi. Convergence of utility indifference prices to the superreplication price: the whole real line case. Acta Applicandae Mathematicae, 96, 119-135, 2007.

[8] L. Carassus, M. Rásonyi. Optimal strategies and utility-based price converge when agents' preferences do. Mathematics of Operations Research, 32, 102-117, 2007.

[9] F. Delbaen, P. Grandits, T. Rheinländer, D. Samperi, M. Schweizer, Ch. Stricker. Exponential hedging and entropic penalties. Math. Finance, 12, 99-123, 2002 .

[10] H. Föllmer, Yu. M. Kabanov. Optional decomposition and Lagrange multipliers. Finance Stoch., 2, 69-81, 1998. 
[11] M. Frittelli, E. Rosazza Gianin. Equivalent formulations of Reasonable Asymptotic Elasticity, Technical Report no. 12, Dept. "Matematica per le Decisioni", University of Florence, 10 pages, 2004. http://newrobin.mat.unimi.it/users/frittelli/publications.html

[12] R. Hodges, K. Neuberger. Optimal replication of contingent claims under transaction costs. Rev. Futures Mkts., 8, 222-239.

[13] Yu. M. Kabanov, Ch. Stricker. On equivalent martingale measures with bounded densities. In:Séminaire de Probabilités, XXXV. Lecture Notes in Math. 1755, 139-148, Springer, Berlin, 2001.

[14] C. Kardaras, G. Žitković. Stability of the utility maximization problem with random endowment in incomplete markets. Preprint. 2008. http://www.ma.utexas. edu/users/gordanz/publications/index.html

[15] D. Kramkov, W. Schachermayer. The condition on the Asymptotic Elasticity of Utility Functions and Optimal Investment in Incomplete Markets. Annals of Applied Probability, 9, 904-950, 1999.

[16] K. Larsen. Continuity of utility-maximization with respect to preferences. Mathematical Finance, 19, 237-250, 2009.

[17] J. Pratt. Risk aversion in the small and in the large. Econometrica, 32, 122-136, 1964.

[18] D. B. Rokhlin. Lower bounds of martingale measure densities in the DalangMorton-Willinger theorem. Preprint. arXiv:0804.1761, 2008.

[19] R. Rouge, N. El Karoui. Pricing via utility maximization and entropy. Math. Finance, 10, 259-276, 2000.

[20] W. Schachermayer. Optimal Investment in Incomplete Markets when Wealth may Become Negative. Annals of Applied Probability, 11, 694-734, 2001. 